American Journal of Immunology 8 (2): 38-43, 2012

ISSN 1553-619X

(C) 2012 Science Publications

\title{
$\beta$ 1, 3-Glucan in Cancer Treatment
}

\author{
Vaclav Vetvicka and J. Vetvickova \\ Department of Pathology, \\ University of Louisville, 511 S. Floyd, Louisville, KY 40202, USA
}

\begin{abstract}
Natural products, useful in preventing and/or treating various diseases, have been sought after throughout the man's history. Despite some problems inherited from the natural source of glucans, there is extensive literature about their activities in animal tumor models. For the past 25 years, Japan has used several forms of mushroom-derived glucans in treating cancer patients. Additionally, close to twenty clinical trials are currently running in several countries, further suggesting the strong possibility of using glucans as an established anti-cancer drug.
\end{abstract}

Key words: Glucan, cancer, immunity, treatment

\section{INTRODUCTION}

$\beta 1,3$-Glucans derived from a variety of natural sources (such as yeasts, grain, mushroom or seaweed) are well-established immunomodulators (sometimes also called biological response modifiers). Numerous reports demonstrated a wide range of biological activities by individual $\beta 1,3$-glucans (for review see Schepetkin and Quinn, 2006; Chen and Seviour, 2007; Novak and Vetvicka, 2008; 2009; Rondanelli et al., 2009; Ramberg et al., 2010; Rahar et al., 2011). Over 10, 000 research papers on the isolation, characterization and biological activities of various glucans exist. Some of them originated from the "golden age" of biological experiments, when authors endlessly kept isolating the same material from new sources. The majority, however, represents a serious science.

The basic glucan research can be summarized in several directions. Isolation and chemical analysis fall outside the scope of this review. The remainder is focused on biological effects. The original studies firmly established the effects on infection immunity (mostly via stimulation of the non-specific branch of immune reactions). Using several experimental models, it has been well established that $\beta$-glucan protects against infection with both bacteria and protozoa and enhances antibiotic efficacy in infections with antibiotic-resistant bacteria. The protective effect of $\beta$ glucans was shown in experimental infection with Leishmania major, Candida albicans, Toxoplasma gondii, Streptococcus suis, Plasmodium berghei, Staphylococcus aureus, Escherichia coli, Mesocestoides corti, Trypanosoma cruzi, Eimeria vermiformis and Eimeria (Cox et al., 2010; for a review see Vetvicka and Novak, 2011). Subsequent experiments returned to the original findings and showed significant support in cancer treatment in both animal and human models (Vetvicka et al., 2002; Hong et al., 2004; Weitberg, 2008).

Animal data: History of polysaccharides as immunomodulators goes back 70 years when Shear and co-workers described a substance, again from Serratia marcescens cultures, that caused necrosis of tumors (Shear et al., 1943). Glucan was later tested in numerous animal models, mostly mouse and rat. The original routes of administration were iv. and ip. Injections, but later attention was also focused on oral administration, with similar results (Hanba and Kuroda, 1988). Among individual types of tumors found to be sensitive to the glucan treatment are lung cancer (Abe et al., 1985), hepatoma (Abe et al., 1984), squamous cell carcinoma (Arika et al., 1986), ovarian cancer (Chen et al., 1991), sarcoma (Diller et al., 1964), prostate cancer (Fullerton et al., 2000), bladder cancer, cervical cancer (Nakano et al., 1996) and breast cancer (Vetvicka and Yvin, 2004).

The mechanisms of glucan's effect on cancer development are still not fully elucidated. The most pronounced and most known effects of glucans consist of augmentation of phagocytosis and proliferative activities of professional phagocytes, such as granulocytes, monocytes and macrophages. These cells recognize and bind glucan via a number of different receptors such as TLR-2 (toll-like receptor 2), Dectin-1, CR3 (complement receptor 3), lactosylceramide and probably others. Binding of glucan to any of the

Corresponding Author: Vaclav Vetvicka, Department of Pathology, University of Louisville, 511 S. Floyd, Louisville, KY 40202, USA Tel: 502-852-1612 Fax: 502-852-1177 
receptors activates macrophages. The activation consists of several interconnected processes including increased chemokinesis, chemotaxis, degranulation, adhesion to the endothelium and migration. In addition, glucan binding also triggers intracellular processes, such as the respiratory burst after phagocytosis of invading cells, increasing activity of hydrolytic and metabolic enzymes and signaling processes leading to activation of other phagocytes and secretion of cytokines and other substances. Persons interested in an excellent review regarding interaction of glucans with macrophages should read (Schepetkin and Quinn, 2006).

The Finding is that glucans bind to the lectin domain of CR3 and prime the receptor for cytotoxic degranulation in response to tumors that bore $\mathrm{iC} 3 \mathrm{~b}$ and were normally resistant to this form of cellular cytotoxicity was key to the successful treatment of cancer. Most solid tumors generate an immune response that results in the deposition of antibody and iC $3 b$ on membrane surfaces. This $\mathrm{iC} 3 \mathrm{~b}$ on tumors serves as a specific target for CR3-bearing neutrophils, macrophages, eosinophils and NK cells that have been primed with soluble $\beta$-glucan (Vetvicka et al., 1996). In addition, when tumors lack such $\mathrm{iC} 3 \mathrm{~b}$, mouse tumor models it has been demonstrated that monoclonal antibodies to tumor antigens can be administered in combination with $\beta$-glucan to restore tumor-bound $\mathrm{iC} 3 \mathrm{~b}$ and assure tumor-specific targeting. Normal tissues surrounding the tumor cells are spared from leukocyte attack because they lack this targeting $\mathrm{iC} 3 \mathrm{~b}$. As a result of these studies, it was only a step away from the current clinical trials using glucans in synergy with commercial anti-tumor molecular antibodies.

Glucan as a drug: In Japan, glucans such as Lentinan derived from the Shiitake mushroom (Lentinula edodes) and Polysaccharide K derived from Coriolus versicolor are approved for use as immunoadjuvants for cancer therapy and have been used for over 20 years (Mansell et al., 1975; Luzio et al., 1980; Morikawa et al., 1985; Chihara et al., 1987). Particular interest is focused on Lentinan which was developed by the Japanese pharmaceutical company Ajinomoto and is now an intravenously administered anti-cancer agent. It is used mainly in patients with advanced/recurrent gastric and colorectal cancers (Taguchi, 1987; Nakano et al., 1999), prostate (Tari et al., 1994) and breast (Kan et al., 1992) carcinoma. Some data obtained on animals suggest the broader possibility of Lentinan action, including acute myeloid leukemia (McCormack et al., 2010). The exact mechanisms are not clear and individual studies suggest $\mathrm{T}$ cell-dependent stimulation of macrophages, switch to Th2-type cytokine production (Hamuro et al., 1999) and activation of NK cells (Amino et al., 1983). For more details on Lentinan and its effect sees (Chihara et al., 1987).

In contrast to other glucans, Lentinan is usually ineffective when used orally. Recently, a superfine dispersed Lentinan with high oral effectivity was introduced. First studies showed not only safety, but significant effects on advanced prostate cancer (Shimizu et al., 2009) and in advanced colorectal cancer (Hazama et al., 2009).

Despite the fact that Lentinan is an official drug, Japanese scientist continue with evaluations of new possible venues of lentinan effects. Advanced oral squamous cell carcinoma is a cancer known for a high resistance to chemotherapy. Lentinan used simultaneously with an oral fluoropyrimidine antineoplastic agent S-1 strongly suppressed the cancer growth, probably via induction of apoptosis (Harada et al., 2010). It seems that, as a drug, Lentinan has a significant future and we can only hope that this anticancer drug will eventually show up in Western medicine as well.

Clinical trials: Since cancer is a leading cause of death worldwide, it is not surprising that most efforts currently focus on evaluating of the effects of glucan on cancer treatment. Several studies showed that low molecular weight, yeast-derived $\beta$-glucan binds to a lectin domain within the $\mathrm{COOH}$-terminal region of the CD11b subunit of complement receptor 3 (CR3, CD11b/CD18 (Xia et al., 1999). Additional studies have indicated that yeast-derived $\beta$-glucans prime neutrophils or natural killer cells for cytotoxicity against iC3b-opsonized tumors via complement activation by anti-tumor antibodies (natural or molecular) (Vetvicka et al., 1997; Yan et al., 1999) Dual ligation of neutrophil CR3 mediated by the Idomain ligand, iC3b and the lectin-like domain ligand by $\beta$-glucans, leads to degranulation and cytotoxic responses (Li et al., 2006). The conclusion of these studies suggests that glucan-mediated tumor immunotherapy utilizes a novel mechanism by which innate immune effector cells are primed to kill iC3bopsonized tumor cells.

Additional reports showed that both soluble and particulate glucans induce proinflammatory cytokine secretion thereby stimulating innate immune effector cell activation. These effects appear to be dependent on Toll-Like Receptor 2 (TLR-2), CR-3 and Dectin-1 pathways (Brown et al., 2003; Gantner et al., 2003; Vetvicka and Yvin, 2004). Our group focused on glucan and CR3 receptors. Subsequent experiments in a variety of murine syngeneic tumors (Yan et al., 1999; Hong et al., 2003; 2004) as well as in human carcinoma 
Am. J. Immunol., 8 (2): 38-43, 2012

xenograft models (Cheung and Modak, 2002; Cheung et al., 2002; Modak et al., 2005; Li et al., 2007; Salvador et al., 2008) revealed the significant therapeutic efficacy of combined glucan and anti-tumor antibody therapy. This concept was confirmed by experiments showing the presence of natural antitumor antibodies in animals and humans and by the necessity of the presence of these antibodies for glucan effects (Yan et al., 1999). As control experiments, this therapy completely fails in CR3$\mathrm{KO}$ mice or in antibody-deficient SCID mice. Later experiments showed that even orally administered glucans retain their therapeutic efficacy in cancer (Nanba and Kuroda, 1987; Cheung and Modak, 2002; Hong et al., 2004).

Several animal studies showed that orally administered glucan significantly augmented cytotoxicity of tumors, but the effects were stronger when the tumors were opsonized with anti-tumor mAb and iC3b. Prelinical studies confirmed that the combination of daily oral doses of glucan and weekly doses of the anti-tumor antibody 14G2a, caused significant tumor regression of $80 \%$ or more compared to treatment with antibodies alone (Hong et al., 2004).

With several thousand scientific papers showing significant effects of glucan therapy in numerous animal models, it is not surprising that significant effort exists in determining if these effects are valid in human patients, too. Currently, there are at least 12 glucan clinical trials in cancer therapy, ranging from Phase I to Phase III (for review Yan, 2011). Most of these trials are based on simultaneous treatment with glucan and specific monoclonal antibodies such as Rutiximax, Avastin or Erbitux. Data from most of these trials have not as yet been released, however a trial proprietary glucan-Imprime PGG plus Erbitux and chemodrug-has released its clinical results and showed that the combination of glucan, Erbitux and Camptosar nearly doubled the overall response rate of second- and thirdline metastatic colorectal cancer patients compared with treatment with individual drugs (Tamayo et al., 2009).

The initial clinical results are both promising and exciting, but without knowing the rest of results, we have to wait for the final judgement on possible use of glucan in cancer therapy.

Glucan as a drug carrier: Lately, glucans found a new role in drug delivery systems either as an actual drug carrier, an adjuvant, or in combination with other materials to form suitable drug delivery systems. Both their immunoadjuvant characteristics and the ability to stabilize drug formulations, facilitate drug delivery and controlled release has been used in drug formulation.
The use of pullulan with water soluble polymers in preparation of ingestible films that can contain pharmaceutical, cosmetic, or biologically active agents can serve as an example. Similarly, nanoparticles based on curdlan were evaluated for potential drug delivery to hepatic carcinoma cells using lactobionic acid as a payload drug ( $\mathrm{Na}$ et al., 2000). Another glucan, schizophyllan, has been extensively studied for use in oligonucleotide delivery (Mochizuki and Saturai, 2009). The Ostroff's group is focused on using glucan particles as a novel drug delivery system. First, they prepared hollow, highly porous microparticles. Second, these whole glucan particles were used as an adjuvant (Adjuvax) with excellent results (Ostroff et al., 1991). In the next step, this group designed several strategies for encapsulation of biologically active material into these microparticles. The results include DNA delivery, siRNA delivery (Soto and Ostroff, 2008) and protein delivery.

Readers keen to get more details on the role of glucan as a carrier of drugs should see an excellent, upto-date review (Soto and Ostroff, 2011).

\section{REFERENCES}

Abe, S., K. Takahashi, J. Tsubouchi, K. Aida and M. Yamazaki et al., 1984. Different local therapeutic effects of various polysaccharides on MH134 hepatoma in mice and its relation to inflammation induced by the polysaccharides. Gann, 75: 459465. PMID: 6745566

Abe, S., K. Takahashi, M. Zamazaki and D. Mizuno, 1985. Complete regression of Lewis lung carcinoma by cyclophosphamide in combination with immunomodulators. Jpn. J. Cancer Res., 76: 626-630. PMID: 3928559

Amino, M., R. Noguchi, J. Yata, J. Matsumura and R. Hirayama et al., 1983. Studies on the effect of lentinan on human immune system. II. In vivo effect on NK activity, MLR induced killer activity and PHA induced blastic response of lymphocytes in cancer patients. Gan. Kagaku Ryoho., 10: 20002006. PMID: 6225393

Arika, T., K. Amemiya and S. Mochizuki, 1986. Combination therapy of radiation and schizophyllan (SPG) in C3H mouse squamous-cell carcinoma NR-S1. Gan. Kagaku Ryoho., 13: 28412847. PMID: 2944485

Brown, G.D., J. Herre, D.L. Williams, J.A. Willment and A.S. Marshall et al., 2003. Dectin-1 mediates the biological effects of beta-glucans. J. Exp. Med., 197: 1119-1124. PMID: 12719478

Chen, J. and R. Seviour, 2007. Medicinal importance of fungal beta-(1-->3), (1-->6)-glucans. Mycol. Res., 111: 635-652. PMID: 17590323 
Chen, J.T., K. Hasumi and K. Masubuchi, 1991. Maintenance of the activation of peritoneal macrophage in patients with ovarian cancer by the repeated administration of sizofiran and interferon gamma. Nippon Sanka Fujinka Gakkai Zasshi, 43: 1599-1605. PMID: 1744455

Cheung, N.K. and S. Modak, 2002. Oral (1-->3),(1-$>4)$-beta-D-glucan synergizes with antiganglioside GD2 monoclonal antibody 3F8 in the therapy of neuroblastoma. Clin. Cancer Res., 8: 1217-1223. PMID: 12006541

Cheung, N.K., S. Modak, A. Vickers and B. Knuckles, 2002. Orally administered beta-glucans enhance anti-tumor effects of monoclonal antibodies. Cancer Immunol. Immunother., 51: 557-564. PMID: 12384807

Chihara, G., J. Hamuro, Y.Y. Maeda, T. Shiio and T. Suga et al., 1987. Antitumor and metastasisinhibitory activities of lentinan as an immunomodulator: An overview. Cancer Detect. Prev. Suppl., 1: 423-443. PMID: 3319150

Cox, C.M., L.H. Sumners, S. Kim, A.P. McElroy and M.R. Bedford et al., 2010. Immune responses to dietary beta-glucan in broiler chicks during an Eimeria challenge. Poult. Sci., 89: 2597-2607. PMID: 21076097

Diller, I.C., M.E. Fisher and D. Gable, 1964. Effect of glucan on mouse sarcoma 37. Proc. Soc. Exp. Biol. Med., 117: 107-110. PMID: 14219916

Fullerton, S.A., A.A. Samadi, D.G. Tortorellis, M.S. Choudhury and C. Mallouh et al., 2000. Induction of apoptosis in human prostatic cancer cells with beta-glucan (Maitake mushroom polysaccharide). Mol. Urol., 4: 7-13. PMID: 10851301

Gantner, B.N., R.M. Simmons, S.J. Canavera, S. Akira and D.M. Underhill, 2003. Collaborative induction of inflammatory responses by dectin-1 and Tolllike receptor 2. J. Exp. Med., 197: 1107-1117. PMID: 12719479

Hamuro, J., Y. Murata, M. Suzuki, F. Takatsuki and T. Suga, 1999. The triggering and healing of tumor stromal inflammatory reactions regulated by oxidative and reductive macrophages. Gann Monograph Canc. Res., 48: 153-164.

Hanba, H. and H. Kuroda, 1988. Potentiation of hostmediated antitumor activity by orally administered mushroom (Agaricus bispora) fruit bodies. Chem. Pharmacol. Bull., 36: 1437-1444. PMID: 3416364

Harada, K., Y. Itashiki, T. Takenawa and Y. Ueyama, 2010. Effects of Lentinan alone and in combination with fluoropyrimidine anticancer agent on growth of human oral squamous cell carcinoma in vitro and in vivo. Int. J. Oncol., 37: 623-631. PMID: 20664931
Hazama, S., S. Watanabe, M. Ohashi, M. Yagi and M. Suzuki et al., 2009. Efficacy of orally administered superfine dispersed lentinan (beta-1,3-glucan) for the treatment of advanced colorectal cancer. Anticanc. Res., 29: 2611-2617. PMID: 19596936

Hong, F., J. Yan, J.T. Baran, D.J. Allendorf and R.D. Hansen et al., 2004. The mechanism by which orally administered beta-1,3-glucans enhance the tumoricidal activity of antitumor monoclonal antibodies in murine tumor models. J. Immunol., 173: 797-806. PMID: 15240666

Hong, F., R.D. Hansen, J. Yan, D.J. Allendorf and J.T. Baran et al., 2003. Beta-glucan functions as an adjuvant for monoclonal antibody immunotherapy by recruiting tumoricidal granulocytes as killer cells. Cancer Res., 63: 9023-9031. PMID: 14695221

Kan, N., H. Kodama, T. Hori, A. Takenaka and T. Yasumura et al., 1992. Intrapleural treatment of breast cancer patients with pleural effusions: an analysis of 13 institutes in Kyoto and Shiga Prefectures. Kyoto and Shiga Breast Cancer Study Group. Gan. Kagaku Ryoho., 19: 1632-1635. PMID: 1530322

Li, B., D.J. Allendorf, R. Hansen, J. Marroquin and C. Ding et al., 2006. Yeast beta-glucan amplifies phagocyte killing of iC3b-opsonized tumor cells via complement receptor 3-Sykphosphatidylinositol 3-kinase pathway. J. Immunol., 177: 1661-1669. PMID: 16849475

Li, B., D.J. Allendorf, R. Hansen, J. Marroquin and D.E. Cramer et al., 2007. Combined yeast \{beta glucan and antitumor monoclonal antibody therapy requires $\mathrm{C} 5 \mathrm{a}$-mediated neutrophil chemotaxis via regulation of decay-accelerating factor CD55. Cancer Res., 67: 7421-7430. PMID: 17671212

Luzio, N.R.D., D.L. Williams, R.B. McNamee and V.G. Malshet, 1980. Comparative evaluation of the tumor inhibitory and antibacterial activity of solubilized and particulate glucan. Recent Results Cancer Res., 75: 165-172. PMID: 7232829

Mansell, P.W., H. Ichinose, R.J. Reed, E.T. Krementz and R. McNamee et al., 1975. Macrophagemediated destruction of human malignant cells in vivo. J. Nat. Canc. Inst., 54: 571-580. PMID: 1123850

McCormack, E., J. Skavland, M. Mjic, O. Bruserud and B.T. Gjertsen, 2010. Lentinan: hematopoietic, immunological and efficacy studies in a syngeneic model of acute myeloid leukemia. Nutr. Cancer, 62: 574-583. PMID: 20574918 
Mochizuki, S. and K. Sakurai, 2009. A novel polysaccharide/polynucleotide complex and its application to bio-functional DNA delivery system. Polymer J., 41: 343-353. DOI: 10.1295/polymj. PJ2008309

Modak, S., G. Koehne, A. Vickers, R.J. O'Reilly and N.K. Cheung, 2005. Rituximab therapy of lymphoma is enhanced by orally administered (1->3), (1-->4) -D-beta-glucan. Leuk. Res., 29: 679683. PMID: 15863209

Morikawa, K., R. Takeda, M. Yamazaki and D. Mizuno, 1985. Induction of tumoricidal activity of polymorphonuclear leukocytes by a linear beta-1,3D-glucan and other immunomodulators in murine cells. Cancer Res., 45: 1496-1501. PMID: 3156669

Na, K., K.H. Park, S.W. Kim and Y.H. Bae, 2000. Selfassembled hydrogel nanoparticles from curdlan derivatives: characterization, anti-cancer drug release and interaction with a hepatoma cell line (HepG2). J. Control Release, 69: 225-236. PMID: 11064130

Nakano, H., K. Namatame, H. Nemoto, H. Motohashi and K. Nishiyama et al., 1999. A multi-institutional prospective study of lentinan in advanced gastric cancer patients with unresectable and recurrent diseases: effect on prolongation of survival and improvement of quality of life. Hepatogastroenterology, 46: 2662-2668. PMID: 10522061

Nakano, T., K. Oka, K. Hanba and S. Morita, 1996. Intratumoral administration of sizofiran activates Langerhans cell and T-cell infiltration in cervical cancer. Clin. Immunol. Immunopathol., 79: 79-86. DOI: $10.1006 /$ Clin. 1996.0053

Nanba, H. and H. Kuroda, 1987. Antitumor mechanisms of orally administered shiitake fruit bodies. Chem. Pharm. Bull. (Tokyo), 35: 24592464. PMID: 3664843

Novak, M. and V. Vetvicka, 2008. Beta-glucans, history and the present: Immunomodulatory aspects and mechanisms of action. J. Immunotoxicol., 5: 47-57. PMID: 18382858

Novak, M. and V. Vetvicka, 2009. Glucans as biological response modifiers. Endocrine Metab. Immune Disorders-Drug Targets, 9: 67-75. PMID: 19275682

Ostroff, G.R., D.D. Easson Jr. and S. Jamas, 1991. A new $\beta$-glucan-based macrophage-targeted adjuvant. ACS Symp. Ser., 469: 52-59. DOI: 10.1021/bk1991-0469. ch006

Rahar, S., Swami, G., Nagpal, N., Nagpal, M.A., G.S. Singh, 2011, Preparation, characterization and biological properties of $\beta$-glucans. J. Adv. Pharm. Technol. Res., 2: 94-103. PMID: 22171300
Ramberg, J.E., E.D. Nelson and R.A. Sinnott, 2010. Immunomodulatory dietary polysaccharides: A systematic review of the literature. Nutr. J., 9: 5454. DOI: 10.1186/1475-2891-9-54

Rondanelli, M., A. Opizzi and F. Monteferrario, 2009. The biological activity of beta-glucans. Minerva Med., 100: 237-245. PMID: 19571787

Salvador, C., B. Li, R. Hansen, D.E. Cramer and M. Kong et al., 2008. Yeast-derived beta-glucan augments the therapeutic efficacy mediated by anti-vascular endothelial growth factor monoclonal antibody in human carcinoma xenograft models. Clin. Cancer Res., 14: 1239-1247. PMID: 18281559

Schepetkin, I.A. and M.T. Quinn, 2006. Botanical polysaccharides: Macrophage immunomodulation and therapeutic potential. Int. Immunopharmacol., 6: 317-333. PMID: 16428067

Shear, M.J., Turner, F.C., A. Perrault and T. Shovelton, 1943. Chemical treatment of tumors. V. Isolation of the hemorrhage-producing fraction from Serratia marcescens (Bacillus prodigiosus) culture filtrates. J. Natl. Cancer Inst., 4: 81-97.

Shimizu, K., S. Watanabe, K. Matsuda, T. Suga and S. Nakazawa et al., 2009. Efficacy of orally administered superfine dispersed lentinan for advanced pancreatic cancer. Hepatogastroenterology, 56: 240-244. PMID: 19453066

Soto, E. and G. Ostroff, 2011. Used of $\beta$-gucans for drug delivery applications. In: Biology and Chemistry of Beta Glucan. Volume 1. $\beta$ GlucansMechanisms of Action, Vetvicka, V. and M. Novak, (Eds). Bentham Science Publishers, pp: 48-67.

Soto, E. and G.R. Ostroff, 2008. Glucan particles as an efficient siRNA delivery vehicle. NSTI Nanotech Tech. Proc., 2: 332-335.

Taguchi, T., 1987. Clinical efficacy of lentinan on patients with stomach cancer: End point results of a four-year follow-up survey. Cancer Detect Prev. Suppl., 1: 333-349. PMID: 3121179

Tamayo, M.E., G.H. Cornelio, J.B. Bautista, M.L. Flores and M.R. Kurman et al., 2009. A phase Ib/2, dose-escalating, safety and efficacy study of imprime PGG, cetuximab and irinotecan in patients with advanced colorectal cancer (CRC). J. Clin. Oncol., 27: e15062-e15062.

Tari, K., I. Satake, K. Nakagomi, K. Ozawa and F. Oowada et al., 1994. Effect of lentinan for advanced prostate carcinoma. Hinyokika Kiyo., 40: 199-123. PMID: 8128920

Vetvicka, V. and J.C. Yvin, 2004. Effects of marine beta-1,3 glucan on immune reactions. Int. Immunopharmacol., 4: 721-730. PMID: 15135314 
Vetvicka, V. and M. Novak, 2011. Biological actions of $\beta$-glucan. In: Biology and Chemistry of Beta Glucan. Volume 1. Beta Glucans-Mechanisms of Action, Vetvicka, V. and M. Novak (Eds.). Bentham Science Publishers, pp: 10-18.

Vetvicka, V., B.P. Thornton and G.D. Ross, 1996. Soluble $\beta$-glucan polysaccharide binding to the lectin site of neutrophil or natural killer cell complement receptor type 3 (CD11b/CD18) generates a primed state of the receptor capable of mediating cytotoxicity of iC3b-opsonized target cells. J. Clin. Invest., 98: 50-61. DOI: 10.1172/JCI118777

Vetvicka, V., B.P. Thornton, T.J. Wieman and G.D. Ross, 1997. Targeting of natural killer cells to mammary carcinoma via naturally occurring tumor cell-bound $\mathrm{iC} 3 \mathrm{~b}$ and beta-glucan-primed CR3 (CD11b/CD18). J. Immunol., 159: 599-605. PMID: 9218574

Vetvicka, V., K. Aterrayama, R. Mandeville, P. Brousseau and B. Kournikakis et al., 2002. Pilot study: Orally-administered yeast $\beta 1$, 3-glucan prophylactically protects against anthrax infection and cancer in mice. J. Am. Nutr. Assoc., 5: 1-5.
Weitberg, A.B., 2008. A phase I/II trial of beta-(1,3) $/(1,6)$ D-glucan in the treatment of patients with advanced malignancies receiving chemotherapy. J. Exp. Clin. Cancer Res., 27: 40-44. PMID: 18803849

Xia, Y., V. Vetvicka, J. Yan, M. Hanikyrova and T. Mayadas et al., 1999. The beta-glucan-binding lectin site of mouse CR3 (CD11b/CD18) and its function in generating a primed state of the receptor that mediates cytotoxic activation in response to iC3b-opsonized target cells. J. Immunol., 162: 2281-2290. PMID: 9973505

Yan, J., 2011. $\quad \beta$-Glucan-mediated tumor immunotherapy-mechanisms of action and perspective. In: Biology and Chemistry of Beta Glucan. Volume 1. $\beta$ Glucans-Mechanisms of Action,. Vetvicka, Novak, V. and M. Bentham Science Publishers, pp: 39-47.

Yan, J., V. Vetvicka, Y. Xia, A. Coxon and M.C. Carroll et al., 1999. Beta-glucan, a "specific" biologic response modifier that uses antibodies to target tumors for cytotoxic recognition by leukocyte complement receptor type 3 (CD11b/CD18). J. Immunol., 163: 3045-3052. PMID: 10477568 